Authors have nothing to disclose with regard to commercial support.

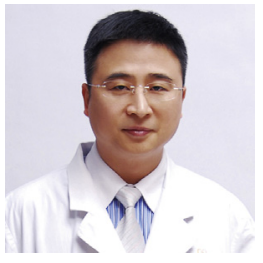

\section{LYMPH NODE DISSECTION DURING SUBLOBAR RESECTION, QUANTITATIVE OR QUALITATIVE?}

\section{To the Editor:}

Two recent articles published in the Journal by Yendamuri and colleagues and Cao and colleagues investigated the effect of the number of lymph nodes (LNs) examined on the survival of patients treated by sublobar resection for early-stage (I/IA) non-small cell lung cancer (NSCLC). ${ }^{1,2}$ Both groups found a positive correlation between LN sampling and prognosis after sublobar resection, suggesting that $\mathrm{LN}$ dissection improves survival outcomes of these patients, even for those who received sublobar resection. According to Yendamuri and colleagues, patients treated by sublobar resection benefitted the most when the number of LNs examined was greater than 9. However, $\mathrm{Hu}$ and colleagues indicated that only 4 LNs require examination to achieve maximum benefit for patients. What may account for this discrepancy?

Examining a larger number of LNs minimizes the risk of overlooking potential metastasis or tumor remnants. Although complete lymphadenectomy is an effective approach for managing risks of metastasis, it is generally not closely followed in practice ${ }^{3}$ and is not necessarily superior to systematic LN sampling, especially for stage IA NSCLC. ${ }^{4,5}$ In fact, systematic LN sampling is the most common choice used by clinicians to surgically treat NSCLC. Thus, a quantitative surrogate is needed that qualitatively indicates sufficient sampling was achieved.

In 2017, our team published an article in the Journal of Clinical Oncology, ${ }^{6}$ in which data from 2 large and representative cohorts, including the US Surveillance, Epidemiology, and End Results database, and a Chinese multi-institutional registry, were evaluated. Specifically, data were analyzed using the hypergeometric distribution

\footnotetext{
The Editor welcomes submissions for possible publication in the Letters to the Editor section that consist of commentary on an article published in the Journal or other relevant issues. Authors should: $\bullet$ Include no more than 500 words of text, three authors, and five references. • Type with double-spacing. • See http://jtcs.ctsnetjournals.org/ misc/ifora.shtml for detailed submission instructions. • Submit the letter electronically via jtcvs.editorialmanager.com. Letters commenting on an article published in the JTCVS will be considered if they are received within 6 weeks of the time the article was published. Authors of the article being commented on will be given an opportunity of offer a timely response ( 2 weeks) to the letter. Authors of letters will be notified that the letter has been received. Unpublished letters cannot be returned.
}

and Bayes theorem, which revealed that sampling at least 16 LNs is sufficient for reliable pathologic node staging and for making accurate prognoses. We know that LN metastasis is determined by the nature of the disease but not the extent of lung resection. In addition, the patients in our study encompassed all operable cases with different stages, pathologies, and surgical procedures. Therefore, the results of our study suggested that 16 is a reliable indicator (ie, surrogate) of the total number of regional LNs that a patient would have and represents a useful cutoff value that ensures sufficient LN sampling.

Several analyses of US population-based databases showed that patients who had undergone sublobar resection had consistently fewer LNs removed than patients who had larger amounts of lung tissue resected. A high percentage of patients $(40 \%$ to $70 \%)$ did not have even $1 \mathrm{LN}$ removed or examined, ${ }^{7,8}$ and this proportion was worse after wedge resection when compared with segmentectomy. ${ }^{9}$ Thus, studies that analyze the effect of number of LN sampling/ dissection should use a large patient dataset that contains patients who received systematic $\mathrm{LN}$ dissection. The studies of Yendamuri and colleagues and $\mathrm{Cao}$ and colleagues both analyzed a specific cohort from the US Surveillance, Epidemiology, and End Results database in which sublobar resection patients had low numbers of LNs examined. The methods used in these studies were based on group stratification rather than trend analyses, which would introduce a significant bias in determining the cutoff number. This is the likely reason why these 2 articles proposed different thresholds for the necessary number of LNs examined. To explore the actual effect of the number of LNs examined in patients with stage IA NSCLC undergoing sublobar resection, we suggest that Yendamuri and colleagues and $\mathrm{Cao}$ and colleagues should have tested 16 LNs examined as the cutoff for sufficient sampling rather than deriving their own cutoff.

However, early noninvasive lung cancer is an exception in the treatment of $\mathrm{LN}$ dissection. It is currently unclear whether lymphadenectomy is necessary for patients with early isolated noninvasive cancer (eg, adenocarcinoma in situ [AIS] or minimally invasive adenocarcinoma [MIA]) that was treated by sublobar resection. In 2015, our group conducted a systematic literature review and found that of 821 patients, only 1 had N1 node metastasis (this 1 patient had MIA); N2 disease was not found in any of the patients. ${ }^{10}$ This indicates that the probability of LN metastasis in AIS/MIA is low. In agreement with this systematic review, previous studies reported 5-year disease-free survival of approximately $100 \%$ in patients with AIS/MIA. Therefore, systematic LN sampling/dissection might be unnecessary for patients with AIS/MIA treated by sublobar resection; however, relevant prospective studies are needed to confirm this.

The US Preventative Services Task Force has released recommendations in favor of computed tomography lung cancer screening for long-term smokers; thus, the incidence 
of early lung cancer will increase markedly. ${ }^{11}$ The impact of lymphadenectomy on patients with early NSCLC after sublobar resection is a critical clinical problem. We propose the following model for LN sampling in patients with NSCLC during sublobar resection: complete systematic LN sampling for invasive carcinomas confirmed by frozen section or uncertain cases, with a threshold of 16 or more LNs being found during postoperative pathologic diagnosis as an indication of sampling sufficiency, but no or limited LN sampling for patients with AIS/MIA when these cancers are intraoperatively confirmed.

Yang Liu, $M D$

Wenhua Liang, $M D, P h D$

Jianxing $\mathrm{He}, \mathrm{MD}, \mathrm{PhD}$

Department of Thoracic Surgery

The First Affiliated Hospital of Guangzhou Medical

University

Guangzhou, China

\section{References}

1. Yendamuri S, Dhillon SS, Groman A, Dy G, Dexter E, Picone A, et al. Effect of the number of lymph nodes examined on the survival of patients with stage I nonsmall cell lung cancer who undergo sublobar resection. J Thorac Cardiovasc Surg. 2018;156:394-402.

2. Cao J, Xu J, He Z, Yuan P, Huang S, Lv W, Hu J. Prognostic impact of lymphadenectomy on outcomes of sublobar resection for stage IA non-small cell lung cancer $</=2 \mathrm{~cm}$. J Thorac Cardiovasc Surg. 2018;156:796-805.e794.

3. Allen JW, Farooq A, O'Brien TF, Osarogiagbon RU. Quality of surgical resection for nonsmall cell lung cancer in a US metropolitan area. Cancer. 2011;117: $134-42$.

4. Darling GE, Allen MS, Decker PA, Ballman K, Malthaner RA, Inculet RI, et al Randomized trial of mediastinal lymph node sampling versus complete lymphadenectomy during pulmonary resection in the patient with N0 or N1 (less than hilar) non-small cell carcinoma: results of the American College of Surgery oncology group Z0030 Trial. J Thorac Cardiovasc Surg. 2011;141:662-70.

5. Huang X, Wang J, Chen Q, Jiang J. Mediastinal lymph node dissection versus mediastinal lymph node sampling for early stage non-small cell lung cancer: a systematic review and meta-analysis. PLoS One. 2014;9: e109979.

6. Liang W, He J, Shen Y, Shen J, He Q, Zhang J, et al. Impact of examined lymph node count on precise staging and long-term survival of resected non-small-cell lung cancer: a population study of the US SEER database and a Chinese multi-institutional registry. J Clin Oncol. 2017;35:1162-70.

7. Kent M, Landreneau R, Mandrekar S, Hillman S, Nichols F, Jones D, et al. Segmentectomy versus wedge resection for non-small cell lung cancer in high-risk operable patients. Ann Thorac Surg. 2013;96:1747-55.

8. Razi SS, John MM, Sainathan S, Stavropoulos C. Sublobar resection is equivalent to lobectomy for T1a non-small cell lung cancer in the elderly: a surveillance, epidemiology, and end results database analysis. J Surg Res. 2016;200: 683-9.

9. Khullar OV, Gillespie T, Nickleach DC, Liu Y, Higgins K, Ramalingam S, et al. Socioeconomic risk factors for long-term mortality after pulmonary resection for lung cancer: an analysis of more than 90,000 patients from the national cancer data base. J Am Coll Surg. 2015;220:156-68.e154.

10. Jiang L, Yin W, Peng G, Wang W, Zhang J, Liu Y, et al. Prognosis and status of lymph node involvement in patients with adenocarcinoma in situ and minimally invasive adenocarcinoma-a systematic literature review and pooled-data analysis. J Thorac Dis. 2015;7:2003-9.

11. Aberle DR, Adams AM, Berg CD, Black WC, Clapp JD, Fagerstrom RM, et al. Reduced lung-cancer mortality with low-dose computed tomographic screening. N Engl J Med. 2011;365:395-409.

https://doi.org/10.1016/j.jtcvs.2018.08.020

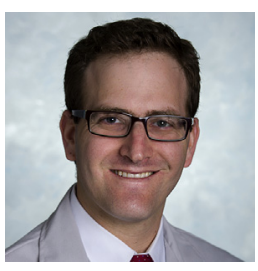

LYMPH NODE

ASSESSMENT

ADEQUACY AND FIRST

AMENDMENT

JURISPRUDENCE: CAN

WE DO BETTER THAN

JUST "KNOWING IT

\section{WHEN WE SEE IT"?}

\section{Reply to the Editor:}

In their letter to the Editor, Liu, Liang, and He highlight an important issue in how we study surgical quality. How do we consistently count lymph nodes, and how to we determine adequacy? To paraphrase Supreme Court Justice Potter Stewart and his ability to recognize obscenity, ${ }^{1}$ we know it when we see it, but we have a very hard time actually quantifying it. This is due in part to (1) fragmentation of lymph nodes during surgery and (2) variation in lymph nodes found within the resected parenchymal specimen. ${ }^{2,3}$ Lymph node count thus becomes an imperfect tool to assess the adequacy of lymph node harvesting. Unfortunately, within large national databases, as of now at least, it is the only tool we have.

The potential benefits of lymph node assessment are both the therapeutic benefit of resecting cancerous nodes and adequately staging disease, especially disease deemed pNO. From the standpoint of staging, adequacy can be determined by assessing the degree of upstaging for a given lymph node strategy (counts, anatomic dissection, number of stations, etc). Liu, Liang, and He describe their method of using hypergeometric distribution and Bayes theorem, by which they identified 16 nodes as a sufficient number. In our group's assessment of the National Cancer Database, ${ }^{4}$ we used a receiver operator curve and sensitivity analysis and found that assessing 14 nodes was sufficient for patients with clinical stage I disease undergoing lobectomy. Several other studies have done similar analyses, and for patients undergoing lobectomy, there seems to be a consensus around 14 to 18 nodes, notwithstanding the concerns already discussed, as sufficient for proper staging.

Patients undergoing sublobar resection are different. As noted in the letter, a large percentage of these patients do not have any lymph nodes assessed. In a study by Yendamuri and colleagues, ${ }^{5}$ only $8 \%$ of patients had more than 9 nodes assessed. In our group's analysis of the National Cancer Database, ${ }^{6}$ only $17 \%$ of patients undergoing wedge resection had more than 5 nodes examined. Neither article specifically said that those were the optimal number of nodes assessed; in fact, given the small number of patients with any nodes assessed, these articles serve to highlight the importance of any node assessment in patients undergoing sublobar resection. Clearly, the lymphadenectomy was 